\title{
La traducción inversa colaborativa como una técnica para mejorar destrezas orales con los estudiantes de un colegio vocacional en Costa Rica
}

\author{
$\ldots \ldots \ldots \ldots \ldots \ldots \ldots \ldots \ldots \ldots \ldots \ldots \ldots \ldots \ldots \ldots \ldots \ldots \ldots \ldots \ldots$ Ruth Cristina Hernández Ching ${ }^{1}$ \& Karen Iliana León Guillén ${ }^{2}$ \\ 1. Docente del Bachillerato en Inglés de la Universidad Nacional, Heredia, Costa Rica \\ ruth.hernandez.ching@una.cr \\ 2. Docente de la Enseñanza del Inglés, Ministerio de Educación Pública, Heredia, Costa Rica \\ karenleon7@gmail.com
}

Recibido: 28 de abril del 2017

Corregido: 19 de junio del 2017

Aceptado: 21 de julio del 2017

\begin{abstract}
Resumen
El artículo presenta los resultados de una exploración sobre el uso de la técnica colaborativa de traducción inversa para mejorar las destrezas orales entre los estudiantes de un colegio técnico vocacional de Costa Rica. Delisle (1998) propone el uso de la traducción pedagógica, mientras que Zhang \& Gao (2014), recomiendan el proceso de negociación entre pares en la enseñanza de segundas lenguas en las aulas de la educación técnica. Se plantea la siguiente pregunta generadora: ¿cómo puede favorecer la técnica de traducción inversa colaborativa en el mejoramiento de las destrezas orales de los estudiantes? Se adopta un método cualitativo de tipo fenomenológico y exploratorio. Se establece como estrategia metodológica la triangulación de resultados en donde se utilizan como instrumentos de medición la observación de las actividades, los grupos focalizados con estudiantes y la entrevista semiestructurada aplicada al profesor del grupo. La población consistió en un grupo de 16 estudiantes de décimo grado. Los resultados de la investigación demostraron que la propuesta permitió el conocimiento de vocabulario nuevo de manera colaborativa, usando material de la traducción profesional por medio de la negociación y el uso de las tecnologías. Estudios posteriores podrían orientarse a medir las ventajas de la técnica para mejorar las competencias comunicativas de segundas lenguas en colegios técnicos y vocacionales.
\end{abstract}

Palabras clave: Pedagogía, traducción, andamiaje, educación técnica, interacción.

\section{Abstract \\ Collaborative back translation as a technique to enhance oral skills with students in a Costa Rica vocational secondary High School}

This paper presents the results while exploring collaborative back translation techniques to enhance oral skills among students in a Costa Rican technical High School. Delisle (1998) proposes pedagogical translation, while Zhang \& Gao (2014), recommend the peer negotiation process in second language teaching. The following generative question is raised: How can vocabulary learning be enhanced by using collaborative back translation to improve oral skills among students? A qualitative approach is adopted. It establishes, as a methodological strategy, the triangulation of results in which the activities' observation, focused to students groups, and semi-structured interview to the the group's teacher are used as measurement instruments. The population of the investigation was based on a group of 16 a $10^{\text {th }}$ graders. Research results demonstrated that the proposal enhanced new vocabulary 
learning by negotiation integrating technologies to produce an adequate learning outcome. Further studies could be oriented to measure the advantages of the technique to improve second language communicative competencies in technical and vocational schools.

Key words: Pedagogy, translation, scaffolding, technical education, interaction.

\section{INTRODUCCIÓN}

En los colegios técnicos y vocacionales en Costa Rica, los mejores niveles de especialización técnica implican un buen manejo de las destrezas en inglés, con el fin de que los estudiantes cuenten con oportunidades de aprendizaje y estándares de calificación apropiados, de ahí la necesidad de crecimiento constante (Álvarez-Galván, 2015). No obstante, de acuerdo con el Ministerio de Educación Pública (2014), existe una carencia de profesores calificados para los programas bilingües, lo cual es uno de los aspectos que afecta la calificación de los estudiantes para que continúen con su ciclo de capacitación.

Los programas de educación vocacional y técnica en Costa Rica emplean como referencia el Marco Común Europeo de Referencia para las Lenguas: Aprendizaje, Enseñanza, Evaluación (MCER, o CEFR, por sus siglas en inglés, Common European Framework of Reference for Languages, 2001), como un estándar para guiar las prácticas en clase. Este proyecto de investigación utiliza la traducción como una técnica para mejorar el aprendizaje de vocabulario nuevo y favorecer la producción oral.

Lejos de la imagen anticuada que se le pueda otorgar a la traducción, como recurso pedagógico, la traducción respalda lo enunciado por Cook (2008) con respecto a los modelos generales del aprendizaje de L2: “La adquisión de una segunda lengua no implica adquirir el sistema de lenguaje autosuficiente de un sistema" (p. 232; traducción de las autoras).

Por otro lado, el proyecto entonces expone la experiencia del uso de la técnica de traducción colaborativa inversa para mejorar las destrezas orales en un colegio técnico público. Para ilustrar la técnica, se utiliza una variación del modelo propuesto por Zhang \& Gao (2014).

\section{REVISIÓN DE LA LITERATURA}

La importancia de la traducción para el aprendizaje de una segunda lengua con fines pedagógicos se puede ilustrar buscando la palabra traducción en el Marco Común Europeo de Referencia para las Lenguas (Consejo Europeo, 2001) se menciona 25 veces. El mismo documento la define como:

El usuario o alumno recibe un fragmento hablado o texto escrito, en una lengua o código determinado $(L x)$, sin que el interlocutor que lo emite esté presente, y produce un fragmento o texto paralelo en una lengua o código diferente (Ly), que será recibido a su vez por otro oyente o lector tampoco presente ( p. 109).

Hernández (1996) define traducción pedagógica como una actividad didáctica con el propósito de mejorar la lengua meta por medio de la manipulación, el análisis contrastivo y la reflexión constante. Delisle (1998) propone una diferenciación entre la traducción pedagógica y la profesional. Se basa principalmente en el establecimiento de relaciones: en el caso de la situación pedagógica, no solo se propone la enseñanza de la detección de ambigüedad o el enfoque basado en la lingüística sino que también la 
observación de los aspectos positivos del texto,; por su parte, en el caso de la traducción profesional, se sugieren las situaciones de formas múltiples y en contacto con el mundo real.

La pregunta que surge es de qué manera la riqueza y diversidad de la traducción profesional puede implementarse en el ambiente educativo. Pintado (2012) afirma que la traducción pedagógica encuentra su razón de ser en un currículo de lenguaje, bajo un paradigma comunicativo y dinámico en donde la enseñanza toma aspectos teóricos y pragmáticos.

Para Zhan y Gao (2014), la traducción inversa, entendida como "la traducción de un texto a la lengua materna (L1) y luego de regreso al inglés" (p. 30; traducción de las autoras), puede, según Douglas y Craig (2007, p. 30) "ofrecer reflexiones acerca de los errores potenciales cuando no se presentaron otros medios disponibles para evaluar la exactitud de la traducción" (p. 30; traducción de las autoras).

La investigación propone una variación de la traducción inversa incorporando la participación colaborativa del estudiante. Zhang \& Gao (2014) definen la traducción como un proceso en donde la entrada o nuevas formas de lenguaje se negocian entre los usuarios usando sus capacidades actuales. Para alcanzarlo, la atención sobre las nuevas formas y el conocimiento previo juega un papel importante. Por ende, los estudiantes se convierten en agentes activos del proceso de aprendizaje, usando el conocimiento previo y aportando mediante la experiencia que adquieren en el proceso de negociación. La siguiente figura muestra esta dinámica de integración de microdestrezas (oral, auditiva, lectora, escrita) por medio de la atención y la negociación:

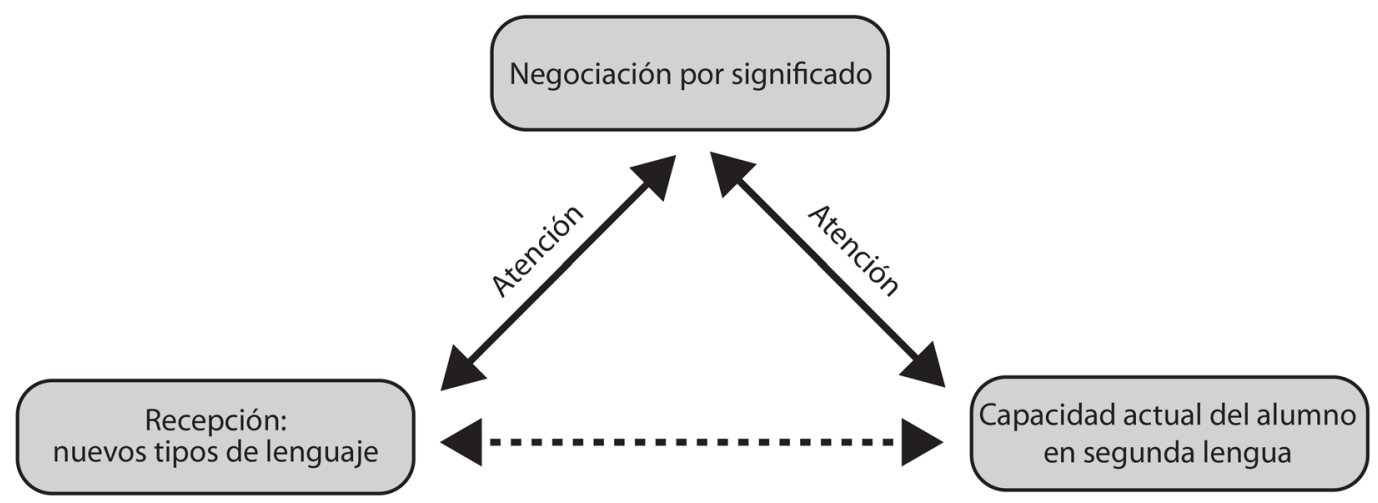

Figura 1. Negociación por significado. Fuente: elaboración propia.

Para Zhang \& Gao (2014), el proceso de andamiaje en el aprendizaje de nuevas lenguas ocurre por medio de la técnica de la traducción inversa, cuando el aprendiz pone atención a contenidos semánticos desconocidos. Es así que, mediante la recepción de nuevos tipos de lenguaje, el estudiante, como sujeto activo y crítico del proceso, utiliza sus capacidades y nota los obstáculos que la tarea traductológica implica. Por medio de la activación del conocimiento previo, la investigación y la negociación, logra superar el obstáculo comunicativo para generar o producir acepciones o significados válidos para el mensaje recibido. La figura se muestra de manera triangular porque es un ciclo constante e integrativo a lo largo de la tarea comunicativa.

Es así como para realizar una propuesta innovadora de la técnica, se decidió que los estudiantes no trabajaran de manera individual, sino de forma grupal, y por consiguiente, el grupo se dividió en subgrupos. Los participantes del colegio público, usando un texto L1 para producir una versión L2 escrita y luego una L1 de manera oral; no obstante, para alcanzarlo, participaron oralmente y negociaron en inglés con sus compañeros para producir una nueva versión L1, tanto de manera escrita como oral. 


\section{DISEÑO DE LA INVESTIGACIÓN}

Este proyecto de investigación, como parte de programa de Licenciatura en la Enseñanza de Segundas Lenguas de la Universidad de Costa Rica, procura buscar soluciones a las dificultades reales diarias que se experimentan en las aulas, o identificar maneras de mejorar los alcances de los estudiantes (Ferrance, 2000). Se aplicó un criterio de conveniencia a la hora de seleccionar la institución. Además, se condujo un enfoque cualitativo para responder la siguiente pregunta generativa: ¿cómo puede favorecer la técnica de traducción inversa colaborativa en el mejoramiento de las destrezas orales de los estudiantes? Para responderla, se programó el siguiente cronograma:

FIGURA 2

Cronograma de actividades.

\begin{tabular}{ll}
\multicolumn{1}{c}{ Actividad } & \multicolumn{1}{c}{ Fecha } \\
Lunes 30 de julio de 2015 & Formulario de Consentimiento Informado (Apéndice 1). \\
Lunes 3 de agosto de 2015 & Reunión con la profesora. Prueba de diagnóstico. \\
Lunes 10 de agosto de 2015 & Aplicación de los instrumentos y recolección de la información. \\
\hline
\end{tabular}

Fuente: elaboración propia.

Así, el día lunes 30 de julio de 2015, se conversó con la docente a cargo del grupo, compuesto por una población finita de 16 estudiantes de décimo año del área técnica Ejecutivo para Centros de Servicio. La profesora propuso realizar una actividad sobre herramientas lingüísticas apropiadas para comunicarse en inglés con otros en la compañía, como lo indica el planeamiento de la lección que se muestra en la Figura 3, particularmente en relación con el objetivo lingüístico de conversación: Asking and responding to questions in clearly defined situations (León, 2015).

Ese mismo día, se realizó una prueba de diagnóstico del libro Business Result (Hughes \& Naughton, 2009) entre los participantes, con el fin de determinar su nivel de inglés. El resultado de la prueba determinó que el grupo poseía un nivel intermedio en las destrezas orales y escritas.

Posteriormente, el día lunes 10 de agosto de 2015, durante el proyecto de investigación, el grupo se organizó en subgrupos de cinco miembros y uno de seis, para completar el experimento en una lección de 40 minutos. Se distribuyó una prueba con 20 preguntas de entrevistas de trabajo en español. Con la guía de la investigadora y la profesora, leyeron las 20 preguntas en voz alta con sus subgrupos. Después de esto, y en inglés, negociaron el significado con los compañeros del subgrupo para producir una versión L2 de manera escrita. En una tabla de Excel $^{\circledR}$ se tabularon los resultados, si producían una versión acertada (1) o no acertada (0). Posteriormente, se les presentó el video en donde se mostraban las preguntas de trabajo de manera escrita y oral. De esta forma, se encontró el espacio para comparar sus versiones de los textos en L2 y L1. Una vez que se concluyó el paso, se les proporcionó la versión original de los textos en español e inglés. Se aplicaron los siguientes instrumentos de medición: observación de los participantes, grupo focal de los participantes y entrevista semiestructurada a la profesora. En el siguiente apartado se plantea una explicación de cada instrumento utilizado.

\section{Observación}

De acuerdo con Creswell (1994), cuando el investigador usa la observación como un participante, él o ella es capaz de registrar la información según se presenta la acción. Todos los participantes conocen el 
FIGURA 3.

Planeamiento de la lección.

Modality: Commerce and Services section. Course: Oral communication in English.

Technical Field: E]ecutivo para Centros de Servicios.

Level: Décimo.

Study Block: Using the Appropriate Linguistc Tools to Communicate in English with others at the Company.

Cognitive Target: Exchanging Information about: Personal Unit $1 \quad$ Hours pertopic: 112 hours. Interaction at the Company, Ways of Interacting, Ethics, Personal skills, Cultural aspects.

\begin{tabular}{|c|c|c|c|c|}
\hline $\begin{array}{l}\text { LINGUISTIC } \\
\text { OBJECTIVES }\end{array}$ & $\begin{array}{l}\text { FUNCTIONS AND } \\
\text { LANGUAGE }\end{array}$ & PROCEDURES & $\begin{array}{l}\text { VALUES AND } \\
\text { ATTITUDES }\end{array}$ & $\begin{array}{c}\text { EVALUATION OF } \\
\text { LEARNING OUTCOMES }\end{array}$ \\
\hline 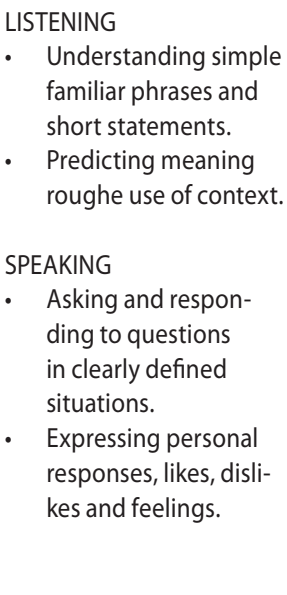 & $\begin{array}{l}\text { FUNCTIONS } \\
\text { - Identifying oneself } \\
\text { to others in the } \\
\text { company. } \\
\text { - Expessing likes. disli- } \\
\text { kes and preferences } \\
\text { and personal qualities } \\
\text { in a professional } \\
\text { environment } \\
\text { - Asking for and giving } \\
\text { information about } \\
\text { personal skills. } \\
\text { Expressing opinions } \\
\text { and concerns. }\end{array}$ & $\begin{array}{l}\text { The students: } \\
\text { - Listen to input } \\
\text { language. } \\
\text { - } \quad \begin{array}{l}\text { Identify basic voca- } \\
\text { bulary from oral and } \\
\text { visual stimuli. }\end{array} \\
\text { - } \quad \text { Perform instructions } \\
\text { given by the teacher o } \\
\text { partners. } \\
\text { - Match meanings with } \\
\text { visual images such as } \\
\text { pictures, drawings and } \\
\text { charts. }\end{array}$ & $\begin{array}{l}\text { Politeness when } \\
\text { dealing with others. } \\
\text { - } \quad \text { Friendliness others. } \\
\text { - Self-respect for others. }\end{array}$ & $\begin{array}{l}\text { The students: } \\
\text { - Imitate words phrases } \\
\text { and sentences through } \\
\text { repetition. } \\
\text { - Exchange greetings, } \\
\text { introductions lea- } \\
\text { ve takings, personal } \\
\text { information. } \\
\text { Demonstrate unders- } \\
\text { tanding of specific and } \\
\text { general information by } \\
\text { using expressions and } \\
\text { role plays on the topic } \\
\text { being s studied. }\end{array}$ \\
\hline
\end{tabular}

Fuente: León (2015).

papel del investigador. Flores y Valenzuela (2012) recomiendan la observación naturalista continua porque es posible entender el contexto y los participantes de una manera no estructurada. Posteriormente, los resultados se reportan usando técnicas de notas extensivas.

En los resultados cualitativos, Miles y Huberman (1994) recomiendan el uso de las fotografías para respaldar la observación y comunicar los resultados. Como una guía para dirigir la investigación, se observaron los siguientes aspectos:

- ¿Los estudiantes negociaron el significado mientras tradujeron?

- ¿ ¿Usaron la comunicación oral para negociar el significado? De ser así, ¿siguieron las estructuras que escucharon en el video?

- ¿La técnica de traducción inversa colaborativa resultó exitosa para generar discurso oral?

\section{Grupo focal con el estudiantado}

El grupo focal es una técnica que le permite al investigador recolectar información planteando preguntas a los participantes y conversando abiertamente sobre sus experiencias con el experimento (Flores y Valenzuela, 2012). Una de las ventajas del grupo focal es que la información se puede obtener de una manera más flexible, sin limitar las respuestas a cuestionarios; de manera que el investigador y los informantes tienen más oportunidades para la interacción, particularmente cuando se cuenta con poco tiempo. 
Se proponen las siguientes preguntas orientadoras para dirigir la discusión con los participantes y el profesor:

- ¿ ¿En qué momentos ha utilizado la traducción para aprender?

- ¿Ha usado la técnica de traducción inversa colaborativa antes?

- ¿Le parece que la técnica de traducción inversa colaborativa es útil para adquirir vocabulario cuando se expresa?

\section{Entrevista semiestructurada a la profesora del grupo}

Una entrevista semiestructurada es una manera de obtener información de una manera flexible y abierta (Flores y Valenzuela, 2012). Las preguntas pretendían determinar si la traducción inversa permitió el aprendizaje de nuevo vocabulario, la colaboración y la producción. Para la entrevista, se utilizaron las siguientes preguntas orientadoras:

- ¿Usa la traducción en sus clases de inglés?

- ¿Usa la traducción inversa en sus lecciones?

- ¿De qué manera la técnica de traducción inversa colaborativa permite mejorar el vocabulario y producir oralmente en la clase?

\section{RESULTADOS}

\section{Observación}

La investigadora completó la observación usando notas extensivas de las visitas a la institución. Se completaron dos de ellas e incluso se incluyeron fotografías para respaldar los instrumentos de observación.

Nota extensiva: La traducción como el medio para comunicarse, no como el fin

Visita \#1

Lugar: Colegio técnico público

Observadora: Ruth Cristina Hernández Ching

Fecha: Lunes 30 de julio de 2015

Hora de inicio: 9:30 a.m.

Hora de finalización: 10:30 a.m.

Hoy se realizó la primera visita a la institución. Después de proporcionarles las instrucciones del proyecto de investigación a los estudiantes, se les brindó una versión L1 del discurso de manera escrita. En grupos, leyeron las oraciones en inglés. Posteriormente, las tradujeron una por una. Si no estaban seguros de la traducción, seguían con la siguiente. Cada miembro ofreció al menos una posible traducción a las oraciones, tanto de manera escrita como oral. Algunos miembros se mostraron más activos que otros; no obstante, no pareció un obstáculo para que todos participaran en la actividad. Los más activos motivaron a los menos activos. En el proceso de traducción, conversaron sobre otros temas en inglés. Al terminar la actividad, el producto final fue una versión escrita del texto propuesto. 
Como interpretación de la primera nota extensiva: La traducción como el medio, no como el fin, se puede señalar que es notorio que los estudiantes se mostraban más cómodos trabajando en equipo. Por otra parte, también estuvieron propensos a participar y menos cohibidos para interactuar. Con respecto a los planteamientos de la teoría de la traducción inversa, aquí se evidencia la negociación de los nuevos términos entre los usuarios, utilizando el conocimiento previo, el cual luego se transforma en conocimiento compartido. Cada subgrupo trabajo de manera independiente, sin la mediación del docente para dicha actividad. Por otra parte, se notó que mientras realizaban el encargo traductológico conversaron de otros temas personales, propiciando otras destrezas del currículo oculto como la colaboración, la tolerancia y la interacción.

\section{Nota extensiva: Diversidad en las traducciones}

Visita \# 2

Lugar: Colegio técnico público

Observadora: Ruth Cristina Hernández Ching

Fecha: Lunes 10 de agosto de 2015

Hora de inicio: 9:20 a.m.

Hora de finalización: 10:20 a.m.

Después de que los tres subgrupos completaron las traducciones, tuvieron la oportunidad de observar el video incluyendo las dos versiones. De manera atenta observaron y escucharon el contenido. En ningún momento se distrajeron porque querían validar la traducción escrita y la pronunciación. Luego, cada grupo tuvo un momento para conversar. Posteriormente, se les entregó una versión impresa de las versiones L1 y L2. Una vez más, compararon los resultados y comentaron sobre la diversidad de traducciones posibles.

Para interpretar la segunda nota extensiva: Diversidad en las traducciones se detecta que, con respecto a la teoría de la traducción inversa y la traducción pedagógica y profesional, se cumplen los postulados por cuando la mediación con material traductológico profesional permitió elevar el nivel de atención del estudiantado, lo cual implica que el proceso resultara significativo. Al mismo tiempo, la actividad vertió luz sobre la riqueza en la traducción puesto que se lograron productos finales diferentes a lo propuesto por el texto original de forma escrita y en el video. En este sentido, al comparar los resultados entre los subgrupos, el estudiantado fue conciente de la variedad lingüística en el proceso comunicativo, manteniendo la fidelidad al encargo.

\section{Grupo focal}

Los segmentos de contenido de los sujetos se categorizaron de acuerdo con las unidades de análisis según la pregunta generadora. A continuación se muestan los datos de la participación del estudiantado en el grupo focal usando tablas descriptivas con su respectiva interpretación, según la teoría:

\begin{tabular}{l|l} 
Traducción inversa & - La técnica resultó útil para conocer el significado y pronunciación de las palabras. \\
para aprender & - Pudimos hacer uso de las ventajas de la tecnología para aprender, como el traductor de \\
& Google y los teléfonos celulares. \\
- También pudimos usar los diccionarios impresos para aprender nuevas palabras. & - Prestamos atención a la pronunciación de palabras escuchando a nuestros \\
compañeros, navegando en la internet, y luego observando el video propuesto. \\
- Además, la técnica realmente nos ayudó para aprender más vocabulario. Otro aspecto \\
importante fue el hecho de que las preguntas pudieran usarse en situaciones \\
de la vida real.
\end{tabular}


Los resultados demostraron que los participantes consideraron que la técnica les permitió aprender el significado y pronunicación de nuevas palabras al superar el obstáculo comunicativo (Zhang y Gao, 2014). Valoraron el aporte de los compañeros, en especial a la hora de aprender la pronunciación de las palabras en un ambiente de cordialidad, respeto y libertad, uno de los aspectos que más valoraran los aprendices con la técnica de la traducción inversa (Zhang y Gao, 2014).

Además, les pareció que la técnica les permitió evacuar significados de manera conjunta pues, cuando alguno no sabía la palabra, otro sí. Llamó la atención también el hecho de valorar el uso de las tecnologías en el proceso de aprendizaje ya que se expresaron de manera positiva a la hora de referirse al uso del video para dicho propósito, así utilizar material de la traducción profesional resultó significativo (Delisle, 1998). Igualmente, valoraron la oportunidad de utilizar diversos recursos tales como el traductor de Google, los teléfonos celulares y los diccionarios impresos.

\begin{tabular}{l|l} 
Traducción inversa & - Cuando no conocíamos el significado de una palabra, otro estudiante lo sabía. \\
para colaborar & - Algunos compañeros tienen una mejor pronunciación también, por lo que podíamos \\
& aprender de ellos. \\
- Logramos negociar significados para proponer una traducción. & Con respecto al video, lo encontramos útil ya que logramos confirmar la \\
& pronunciación de las palabras.
\end{tabular}

Con respeto al postulado teórico de la traducción inversa sobre la negociación por significado (Zhang \& Gao, 2014), los participantes expresaron que lograron aprender mejor colaborando con otros, pues si alguno no conocía el significado de otra palabra, otro sí lo sabía. Al mismo tiempo, rescataron la posibilidad de negociar con los pares para llegar a un significado acorde al gusto y estilo de todos los miembros. Por lo tanto, la capacidad actual del alumno en segunda lengua jugó un papel valioso en el proceso. La observadora realizó la verificación de los textos metas y corroboró que el mensaje recibido y enviado fuera aceptable.

\begin{tabular}{l|l} 
Traducción inversa & - Fuimos capaces de proponer una traducción de todas las preguntas. \\
para producir & - Logramos confirmar el significado de las palabras usando la experiencia y las \\
& contribuciones de nuestros compañeros y el video.
\end{tabular}

Esta última categorización resulta vital ya que se demuestra que la técnica propuesta permite alcanzar el objetivo principal de aprendizaje que busca el Marco Común Europeo al incluir la traducción en los procesos de enseñanza en donde el estudiante produce un texto paralelo de un hablante o escritor, utilizando un lenguaje o código diferente (Common European Framework of Reference for Languages, 2001, p. 108). Los estudiantes manifestaron su satisfacción al lograr vertir una versión completa del texto. Por otra parte, lograron confirmar el significado de las palabras con la ayuda del video o la versión impresa que se les proporcionó. Asimismo, valoraron la tecnología como un recurso para producir de manera oral y escrita.

\section{Entrevista semiestructurada}

Los resultados de la entrevista semiestructurada realizada a la persona docente también se categorizaron en segmentos de contenido basados en las unidades de análisis según las preguntas generadoras: ¿usa la traducción en sus clases?, ¿usa la traducción inversa en sus lecciones?, ¿de qué manera la técnica de la traducción inversa colaborativa permite mejorar el vocabulario y producir oralmente en la clase? A continuación, se muestra la información en las siguientas tablas descriptivas: 


\begin{tabular}{l|l} 
Traducción inversa & - La técnica se ha utilizado previamente en muchas lecciones pero principalmente para \\
para aprender & introducir nuevo vocabulario. \\
- Les ofrecía a mis estudiantes listas de vocabulario para que lo tradujeran. \\
- Usaban diccionarios impresos o electrónicos para traducir.
\end{tabular}

Con respecto al uso de la técnica en el aula, se ha utilizado principalmente para traducir listas de vocabulario (método gramatical), no en contexto y de manera individual. Además, el uso de los recursos tecnológicos y material impreso se ha utilizado principalmente para aprender vocabulario. Por lo tanto, si bien es cierto que los estudiantes demostraron un nivel aceptable de competencia en el idioma inglés, existe una oportunidad de mejora, a la luz de la teoría y según los resultados de este experimento, de implementar prácticas novedosas en la clase. Con respecto al uso de la tecnología, se detecta la oportunidad de utilizarla de manera integrativa en el proceso de aprendizaje.

Traducción inversa para colaborar
- No usé la traducción inversa porque tomó mucho tiempo para preparar las lecciones usando esta técnica, sin embargo, a los estudiantes les encanta traducir y trabajar de manera colaborativa.

De la entrevista se desprende que la docente considera que el planificar actividades de este tipo quizás demanda más tiempo, aunque en esta ocasión resultó significativo y pertinente debido a que se propuso como una posible tarea futura, es decir, una entrevista de trabajo. Lo anterior respalda el postulado teórico de Delisle (1998). Por otra parte, también se respalda lo enunciado por Zhang \& Gao (2014), pues se vislumbra la traducción inversa como un triángulo integrativo de autogestión, cooperación e integración de microdestrezas.

\begin{tabular}{l|l} 
Traducción inversa & - Considero que la técnica funciona mejor si trabajamos con segmentos. \\
para producir & - He usado la traducción inversa pero sin considerarla una técnica. \\
- De acuerdo con esta experiencia, pude observar que a los estudiantes les gusta \\
traducir, especialmente cuando se les ofreció material que les puede servir para \\
realizar tareas futuras, como presentarse a una entrevista de trabajo. \\
- Me gustó la técnica. Lo único es que toma más tiempo preparar las lecciones.
\end{tabular}

Según lo expresado por la docente, la técnica aquí propuesta permitió la exploración e integración de capacidades para obtener un producto final paralelo a partir de la recepción de nuevos tipos de lenguaje, utilizando las capacidades del alumno en segunda lengua para negociar un significado aceptable Zhang \& Gao (2014), por medio de la activación de la atención (aprendizaje significativo), la colaboración y la integración, en conjunto con los recursos tecnológicos.

\section{CONCLUSIONES}

Los datos de la investigación realizada respaldan lo enunciado por Delisle (1998) por cuanto la traducción pedagógica ofrece a la experiencia docente riqueza y diversidad; el bagaje profesional puede implementarse en el ambiente educativo, lo que Pintado (2012) denomina la combinación de aspectos teóricos y pragmáticos. Los resultados respaldan la afirmación anterior cuando tanto los estudiantes como la docente afirmaron que la técnica les resultó significativa ya que se trabajo con una entrevista de trabajo, es decir, una situación real. 
Por otra parte, la docente manifestó el uso que le había dado a la técnica, principalmente relacionado a un enfoque del método gramatical de la traducción (Richards \& Rodgers, 2001), por ende, de la enseñanza de una segunda lengua.

Por tanto, se puede concluir que la mediación pedagógica con la técnica de interpretación inversa facilitó, según Gutiérrez y Prieto (1999): “El tratamiento de contenidos y de las formas de expresión de los diferentes temas a fin de hacer posible el acto educativo, dentro del horizonte de una educación concebida como participación, creatividad, expresividad y relacionalidad" (p. 9). Esto lo respaldan las afirmaciones de los estudiantes cuando encontraron participación, apoyo, libertad y cordialidad en sus compañeros a la hora de realizar la actividad por medio del uso de un aprendizaje significativo pues sabían que el tipo de ejercicios los podía transportar a la cotidianidad en un futuro cercano.

De la misma forma, los estudiantes confirmaron con sus compañeros si la traducción existía previamente en sus repertorios; por ende, aplicaron el conocimiento previo para solucionar la actividad, como lo propone la técnica propuesta por Zhang \& Gao (2014). Si no lo sabían, entonces consultaban a otros compañeros, consultaron los recursos impresos o electrónicos, y finalmente, consultaron el video. La profesora no medió cuando necesitaban conocer significados, por lo cual la técnica promovió el aprendizaje autónomo.

Al usar la técnica de traducción inversa, lograron negociar el significado y ofrecer productos integrados: dicho, escrito, escuchado, leído. Las diferentes fuentes de significado se convirtieron en parte del proceso de traducción pedagógica como el conocimiento previo y el trabajo colaborativo. Los estudiantes utilizaron técnicas integrativas para producir una versión aceptable del discurso. Por otra parte, la tecnología se convirtió en un miembro auxiliar de cada subgrupo.

Por estos motivos, se puede afirmar que el proyecto de investigación acción cumplió con lo que indica el Marco Común Europeo de Referencia para las Lenguas (Consejo Europeo, 2001):

De igual modo, un escolar que tiene que traducir un texto de una lengua extranjera (tarea) puede ver si ya existe una traducción, puede pedir a un compañero que le enseñe lo que ha hecho, puede utilizar un diccionario, puede intentar deducir algún significado basándose en las pocas palabras o estructuras que conoce, puede discurrir una buena excusa para no presentar este ejercicio, etc. (todas las posibles estrategias). Para todos los casos previstos aquí, habrá necesariamente una actividad de lengua y un procesamiento de texto (traducción o mediación, negociación verbal con un compañero de clase, excusas escritas o verbales dirigidas al profesor, etc.) (p. 29).

La apropiación de vocabulario entonces se convirtió en una experiencia de atención, conocimiento, desconocimiento, activación de conocimiento previo, inferencias, adivinación de significados, interacción con otros, uso de la tecnología como una herramienta, uso de la imaginación, negociación de significados y producción integrada. Para los estudiantes resultó posible imaginar un escenario laboral en donde interpretaban contenido real.

La traducción del discurso pasó a un segundo plano en donde más bien los estudiantes contaron con un espacio de interacción el cual Zhang \& Gao (2014) proponen como una forma de captar la atención en el experimento. La experiencia promovió una cultura de enseñanza, negociación y colaboración. La traducción inversa, por lo tanto, y en este particular, la traducción inversa pedagógica, permitió a los estudiantes trabajar de manera proactiva, motivados para escribir y hablar.

En este caso, la incorporación de la tecnología utilizando el video y el audio resultó un recurso pertinente, junto con las destrezas de traducción, para motivar en los estudiantes el proceso de aprendizaje de vocabulario. De hecho, la hablante del video se convirtió virtualmente en parte del escenario de aprendizaje. Lo anterior, según Slimani y Williams (en Dekeyser, 2007) "los aprendices se muestran más perceptivos al lenguaje en intercambios que inician sus compañeros" (p. 183; traducción de las autoras). 
Con respecto a algunas recomendaciones para aquellos que deseen replica esta experiencia, hubiese sido importante realizar una evaluación posterior, fuera sumativa, formativa o alternativa para determinar cuán significativa resultó la técnica en términos de conocimiento de nuevo vocabulario.

Por otra parte, para el grupo la actividad no presentó un mayor desafío pues de acuerdo con la prueba de diagnóstico, contaban con un nivel B2. No obstante, sí resultó innovador el hecho de realizarlo con una situación comunicativa real y no la traducción de listas de vocabulario.

Además, en los instrumentos se pudo incluir una pregunta para determinar el tipo de material que utilizaban para interpretar según lo recomendado por Delisle (1998). Zhang \& Gao (2014), también recomiendan el estudio posterior de la diversidad en la versiones traducidas incorporando el análisis de la diferencia en la escogencia de palabras (el léxico), así como los elementos culturales envueltos en el triángulo o círculo traductológico-pedagógico-profesional.

La enseñanza por medio de la traducción pedagógica es un área de conocimiento que puede contribuir enormemente en el proceso de adquisición de una segunda lengua. Los profesores se beneficiarían al recibir capacitación adicional en esta material. Asimismo, estudios posteriores podrían orientarse a medir las ventajas de la técnica para mejorar las competencias orales y escritas durante un período prolongado de tiempo.

\section{REFERENCIAS}

Álvarez-Galván, J. (2015), A Skills beyond School Review of Costa Rica, OECD Reviews of Vocational Education and Training, OECD Publishing, Paris. Recuperado de: http://dx.doi.org/10.1787/9789264233256-en

Cook, V. (2008). Second Language Learning and Language Teaching (Fourth ed.). London: Hodder Education.

Consejo Europeo (2001). Marco Común Europeo de Referencia para las Lenguas: Aprendizaje, Enseñanza, Evaluación. Recuperado de: https://cvc.cervantes.es/ensenanza/biblioteca_ele/marco/ cvc_mer.pdf

Creswell, J. (1994). Diseño de investigación. Aproximaciones cualitativas y cuantitativas. California: Sage Publications.

Dekeyser, R. (2007). Practice in Second Language (First ed.). New York: Cambridge University Press.

Delisle, J. (1988). Translation: An Interpretative Approach. Ottawa: University of Ottawa Press.

Douglas, S. \& Craig, S. (2007). Collaborative and Iterative Translation: An Alternative Approach to Back Translation. Journal of International Marketing, 15(1), 30-43. Recuperado de:

http://journals.ama.org/doi/abs/10.1509/jimk.15.1.030?journalCode=jimk

Ferrance, E. (2000). Action Research. Richmond: Brown University.

Flores, M. y Valenzuela, J. (2012). Fundamentos de Investigación Educativa. México: Editorial Digital Tecnológico de Monterrey.

Gutiérrez, F. y Prieto, D. (1999). La Mediación Pedagógica. Buenos Aires: Ediciones Ciccus.

Hernández, R. (1996). La traducción pedagógica en la clase de E/LE. Recuperado de: http://cvc.cervantes.es/ensenanza/biblioteca_ele/asele/pdf/07/07_0247.pdf

Hernández, R. (2011). El proceso de enseñanza-aprendizaje de la traducción: comparación de tres actividades en presencia y ausencia de herramientas informáticas (Trabajo de graduación para aspirar al grado de Magíster en Traducción Inglés-Español). Universidad Nacional, Heredia.

Hughes, J. \& Naughton, J. (2009). Business Result. Intermediate. Oxford: Oxford University Press. 
León, K. (2015). 10th Grade English Class Planning. Heredia: Colegio Técnico de San Pedro, Barva.

Miles, M. \& Huberman, A. (1994). Qualitative data analysis. Thousand Oaks: Sage.

Pintado, L. (2012). Fundamentos de la traducción pedagógica: traducción, pedagogía y comunicación. SENDEBAR, 23, 321-353.

Richards, J. \& Rodgers, T. (2001). Approaches and methods in language teaching. New York: Cambridge University Press.

Zhang, Y. \& Gao, C. (2014). Back Translating: An Integrated Approach to Focus Learners'Attention on Their L2 Knowledge Gaps. English Teaching Forum, 52(1), 30-35. Recuperado de: http://files.eric.ed. gov/fulltext/EJ1029177.pdf 